\title{
Arquitecturas híbridas para el diseño de prácticas docentes con recursos online: un caso de studio
}

\author{
Guillermo Castilla Alcaláa ${ }^{a}$ Jose Ignacio Ortiz González ${ }^{\text {b }}$, Alfonso Durán Heras ${ }^{c}$ \\ Escuela Politécnica Superior de la Universidad Carlos III de Madrid, España, \\ acastilla@ing.uc3m.es, bjoortiz@ing.uc3m.es, cduran@ing.uc3m.es
}

\section{Resumen}

La enseñanza de Sistemas de Información Gerencial (SIG) precisa una metodología activa en la que el alumno utilice y experimente con estos sistemas en entornos similares a la realidad empresarial.

Ahora bien, el diseño y desarrollo de prácticas de laboratorio con estos sistemas exige como prerrequisito disponer del correspondiente software, cargado con un conjunto de datos apropiado.

En los últimos años, el auge de los sistemas de código abierto y el movimiento hacia la computación en la nube, hacen habitual, por una parte, encontrar en internet "demos" online de libre acceso, o sistemas de prueba con distintos grados de usabilidad, de aplicaciones empresariales. Por otra parte, los sistemas de código abierto permiten, si se dispone de los conocimientos y las plataformas hardware adecuadas, su descarga, instalación y configuración en modo local sin incurrir en coste de licencias.

Esto abre un abanico de opciones en cuanto a la arquitectura a utilizar para el diseño de actividades educativas basadas en aplicaciones software, con diferentes ventajas, inconvenientes y requerimientos de recursos.

Desde el simple acceso en modo visualización a "demos" existentes en la nube a la descarga y personalización del software en local, pasando por opciones híbridas que combinan estos enfoques en la misma práctica docente.

Así, en este trabajo se presentan posibles arquitecturas híbridas para la implantación de actividades docentes de prácticas de laboratorio basadas en la combinación de tareas realizadas en sistemas de libre acceso en la nube y en sistemas implantados localmente.

Estos conceptos se ilustran con una experiencia de aplicación de estas arquitecturas híbridas en el diseño de una práctica de laboratorio de la 
Universidad Carlos III de Madrid basada en aplicaciones de código abierto. Esta experiencia combina un entorno implantado localmente y aplicaciones online en la nube, partiendo del mismo juego de datos en ambos entornos.

Palabras claves: Prácticas docentes, arquitecturas, Sistemas de Información Gerencial, Odoo.

\section{Introducción}

Hoy en día pocos dudan de la importancia de las actividades prácticas docentes con ordenador que promueven el aprendizaje activo en la enseñanza. El aprendizaje basado en actividades prácticas constituye un pilar del modelo de enseñanza actual en la educación superior. Los procesos de aprendizaje en el área de ingeniería, un área de la ciencia eminentemente práctica, precisan contar con actividades educativas que permitan la experimentación y la práctica en entornos similares a la realidad empresarial.

Si además se trata de enseñar Sistemas de Información Gerencial (SIG) a futuros ingenieros, también denominados MIS por sus siglas en inglés Management Information Systems, se hace imprescindible plantear prácticas de formación online donde el alumno utilice dichos sistemas en distintos escenarios empresariales.

La tendencia de avance en la adopción de las tecnologías de la información y comunicaciones (TIC) en la educación superior confirma que los estudiantes son cada vez más hábiles en relación a los entornos digitales y más favorables a que la tecnología y formación online ocupe parte de la formación presencial (Adams Becker, S. Cummins, Davis, Freeman, Hall Giesinger, \& Ananthanarayanan, 2017).

Las teorías del aprendizaje constructivistas se apoyan en el aprendizaje activo, en el que el aprendizaje consiste principalmente en la construcción de la estructura de conocimiento y posterior refinamiento en la mente del alumno. Las clases en las que los estudiantes construyen su propio aprendizaje son más eficientes en la formación de las actitudes y habilidades que el ingeniero precisa para el desempeño de su profesión (Caro \& Reyes, 2003).

En el área de Sistemas de Información para la Gestión empresarial (SIG), este aprendizaje activo se lleva a cabo mediante actividades diseñadas por el docente sobre los propios sistemas, en base a la construcción de entornos simulados y juegos que desarrollan capacidades de manejo y análisis de la información. De este modo, se plantean escenarios del mundo empresarial en los que el alumno ha de enfrentarse a problemas de gestión y toma de decisiones, que posteriormente son objeto de debate en relación al uso de la 
información o sobre los que realizan trabajos de análisis crítico sobre las problemas y situaciones planteadas. De este modo, el alumno construye su propio conocimiento sobre la utilidad de los Sistemas de Gestión.

La enseñanza con apoyo de ejercicios prácticos basados en TIC tiene claras ventajas para los alumnos (Marcos Ortega, de Borja Varona, \& Manuel Lopez, 2013).

Para plantear la enseñanza como un proceso de descubrimiento y entendimiento por parte del alumno, es fundamental basar el aprendizaje en la realización de ejercicios prácticos que sumerjan gradualmente al alumno en la lógica de dichos sistemas.

Diseñar ejercicios prácticos basados en el uso de la tecnología informática constituye una tarea compleja que requiere tener muy presentes los objetivos docentes.

Según los modelos de diseño instruccional, como el RASE (Churchill, King, \& Fox, 2013), se precisa disponer de recursos para diseñar actividades educativas que motiven e involucren alumno. Con estos recursos han de definirse las actividades que posteriormente puedan ser evaluadas para determinar el progreso del aprendizaje y aseguren que el alumno consigue los objetivos docentes planteados.

El diseñador instruccional requiere disponer de las aplicaciones y los conocimientos sobre las mismas que le permitan adaptarlas a los objetivos docentes. Los conocimientos que se requiere son tanto a nivel informático-técnico como a nivel de características y funcionalidades de las aplicaciones. El docente fija los objetivos docentes que quiere transmitir y ha de tener la capacidad para utilizar y manejar el software específico para crear entornos simulados, en los que el alumno pueda experimentar con las aplicaciones y valorar las ventajas e inconvenientes del empleo de las distintas funcionalidades del software.

El diseño de unas prácticas educativas basadas en SIG, adecuadas a los contenidos curriculares, es un proceso complejo y multidisciplinar que requiere tener acceso a los sistemas de información y a los datos correspondientes. Estos datos han de ser adecuados y en consonancia con el escenario simulado sobre el que se plantea la actividad educativa.

Existe gran variedad de tipos de sistemas de información sobre los que se puede plantear docencia: sistemas de información para la gerencia, sistemas de organización industrial, sistemas productivos y logísticos, de gestión de calidad, de gestión de proyectos, sistemas de información geográficos, sistemas de comercio electrónico (eCommerce), bases de datos especializadas, etc. Muchos de estos sistemas son componentes de los llamados ERP (Enterprise Resource Planning), CRM (Customer Relationship Management), ECM (Enterprise Content Management) o BIS (Business Intelligence Systems). 
No siempre es fácil disponer de estos sistemas software y de juegos de datos para diseñar y desarrollar prácticas de laboratorio en las que el alumno pueda acceder a las aplicaciones de gestión empresarial en entornos similares a situaciones de la vida real.

En los últimos años, el auge del código abierto ha incrementado la disponibilidad y calidad de aplicaciones de carácter libre gratuito disponibles para ser descargadas e instaladas en un ordenador. Adicionalmente, el movimiento hacia la computación en la nube hace que, en muchas ocasiones, sin necesidad de descarga e instalación, sea habitual encontrar en internet "demos" online, o sistemas de prueba con distintos grados de usabilidad, para distintos ámbitos empresariales, libremente accesibles en las correspondientes nubes.

Muchas instituciones han creado repositorios y portales con material educativo que incluye herramientas software y plataformas on-line creadas con intencionalidad educativa (González Ruiz, Martín Gómez, \& Vega Navarro, 2018).

Todo esto posibilita la compartición y adaptación del software para la construcción de actividades docentes, sin que esto suponga coste de licencias de uso (Ecology \& For, 2017).

\section{Arquitecturas}

La enorme disponibilidad de recursos de internet abre un abanico de opciones en cuanto a la arquitectura a utilizar para el diseño de actividades educativas basadas en aplicaciones software con diferentes ventajas e inconvenientes.

Así, en un extremo estaría el simple acceso en modo visualización a "demos" existentes en la nube, donde el alumno tiene una capacidad muy limitada de interactuación, y los datos están total o parcialmente predefinidos, lo que dificulta alcanzar los objetivos docentes. Así mismo, estos datos predefinidos pueden ser inicialmente compartidos si, por ejemplo, los alumnos acceden con el mismo usuario (login), o pueden ser datos independientes para cada alumno, aunque estos sean inicialmente iguales para todos, en cuyo caso cada uno tendrá una copia de los mismos datos y podrá modificarlos sin alterar los datos del resto.

En el extremo opuesto, estaría la instalación completamente en local (incluso, en el caso más extremo, instalando determinados módulos o componentes o incluso habiendo modificado parcialmente el código descargado con desarrollos a medida), parametrizada y cargada con datos específicamente generados para alcanzar dichos objetivos. Este extremo requiere el uso intensivo de recursos y exige conocimientos, dedicación y esfuerzo. En este caso se abre la posibilidad a dar al alumno los privilegios que se estimaran oportunos para las actividades que debiera realizar: uso, parametrización, instalaciones, accesos directos a los datos y extracciones a otros sistemas, etc. Este tipo de entornos pueden ajustarse 
completamente a los objetivos de aprendizaje, pero requiere disponer de suficientes recursos informáticos, tiempo y conocimiento a nivel local.

Entre estos extremos se sitúan las alternativas intermedias con distintos juegos de datos de prueba personalizados o replicando datos predefinidos del software de la nube en entornos locales. Estas opciones híbridas permiten combinar distintos enfoques en la misma práctica o en un conjunto integrado de éstas, para explotar óptimamente las fortalezas relativas de cada uno.

Como no siempre los datos y aplicaciones disponibles en la nube se adaptan a los objetivos docentes, se precisa una adaptación y personalización de los mismos. Dado que el docente no es el propietario de los sistemas en la nube, no es fácil y a veces inviable, la adaptación de los datos y aplicaciones. Los sistemas disponibles en la nube están diseñados para otros fines, fines comerciales principalmente, que permiten comprobar la calidad de los mismos, alejados del enfoque didáctico que el docente precisa.

Con actividades docentes en entornos híbridos que combinan el acceso a sistemas en la nube y sistemas en local, el alumno no solo practicará con sistemas de información gerencial, sino que también se habituará a trabajar con entornos híbridos, cada vez más comunes en la empresa.

Con objeto de explorar el abanico de arquitecturas para la implantación de actividades prácticas de laboratorio sobre SIG disponibles en internet se plantea un mapa en base a dos dimensiones.

Una primera dimensión determinada por la localización de las aplicaciones y datos (local/nube), y una segunda dimensión con el grado de personalización de estos.

En los entornos de la nube el docente tiene poco control y es difícil que tenga acceso a personalizar los datos y aplicaciones. En la mayoría de los casos el entorno en la nube se ajustará de forma parcial a los objetivos del docente. En los entornos en local el grado de personalización es total.

Las dimensiones mencionadas generan un espacio de arquitecturas híbridas para el diseño de las actividades prácticas formativas basada en aplicaciones libres donde se combinan sistemas en local y en la nube, tal como se muestra en el esquema de la Fig. 1. 


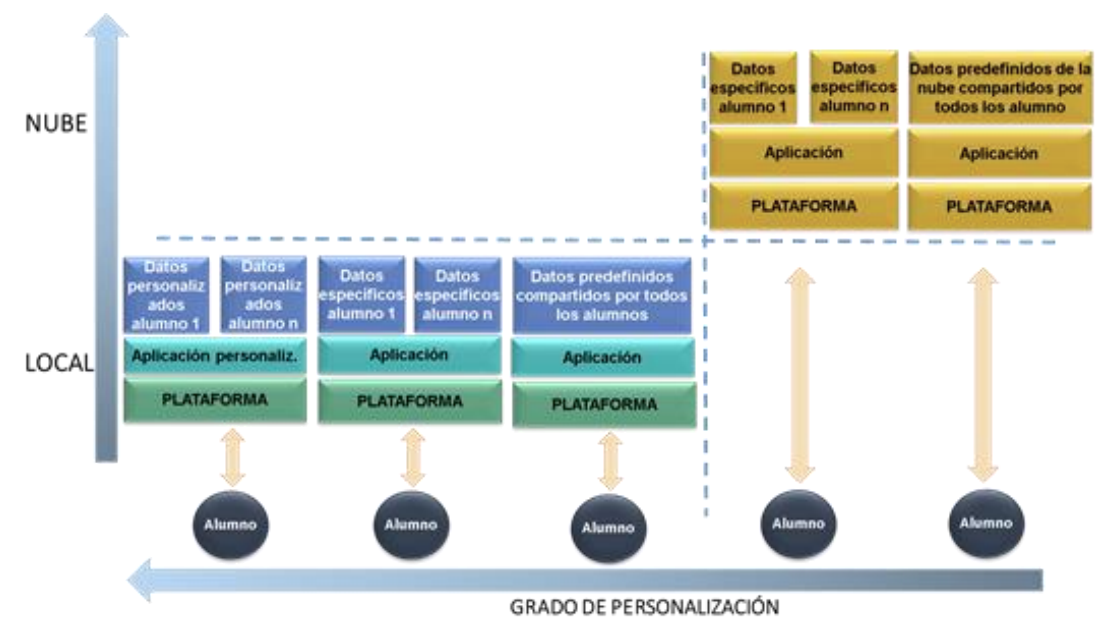

Fig. 1 Arquitecturas. Fuente: elaboración propia

Entre las dos situaciones extremas, el uso de demos de terceros en la nube y el uso de sistemas instalados localmente, es posible encontrar puntos intermedios híbridos que reducen el esfuerzo de la implantación local combinando el uso de dicha implantación local para ciertas tareas con la utilización de determinadas tareas disponibles de forma genérica en la nube que posibiliten la personalización de las actividades formativas adaptadas a los objetivos docentes.

Los cuadrantes de la derecha corresponderían a las aplicaciones (demos) que ofrecen algunos fabricantes de SIG (software de código abierto o propietario) a modo de pruebas gratuitas (por ejemplo, Odoo SA, 2019; SAP AG, 2019) que permiten acceso gratuito a los distintos componentes de las aplicaciones en la nube.

En el caso de demos en la nube, es habitual que contengan precargados unos datos de ejemplo, que llamamos "datos específicos" o "predefinidos". Estos datos, en algunos casos pueden personalizarse con datos que interese al docente y en otras ocasiones no es posible. En los sistemas implantados en local siempre será posible cargar los datos personalizados que se dispongan, incluso se podrán carga los datos específicos de las demos de la nube. Así mismo, en los sistemas implantados en local siempre será posible la instalación de los módulos adecuados, o combinarlos con otras aplicaciones o herramientas como en el caso que se comenta en la próxima sección. 


\section{Caso de estudio}

Se presenta una experiencia de aplicación de una arquitectura híbrida utilizada en el diseño de una práctica de laboratorio de la asignatura de Dirección y Sistemas de Información del Master en Ingeniería Industrial de la Universidad Carlos III de Madrid basada en aplicaciones de código abierto.

Los objetivos docentes que se plantean son, en primer lugar, comprender que la capacidad de integración de un ERP reside en su base de datos y su modelo de datos compartido por todos los módulos funcionales, y en segundo lugar, experimentar la conversión de datos en 'información y conocimiento' mediante la elaboración de informes a medida a partir de datos transaccionales. Se trata de que el alumno tenga una experiencia de acceso al Sistema de Gestión de Base de Datos (SGBD) de un ERP que almacena los datos operativos transaccionales, con la posibilidad de elaborar informes personalizados nivel táctico a partir de los datos almacenados en tablas de dicho SGBD, a diferencia de los informes predefinidos que el ERP pueda proporcionar.

En este caso se utilizó un ERP de código abierto Odoo, el cual se descargó gratuitamente, se instaló en una plataforma local y se configuró una "instancia" con datos personalizados sobre productos, proveedores, inventarios, clientes, pedidos de venta y de compra etc.

Con el nombre de "instancia" nos referimos de forma genérica, a lo que en los sistemas ERP como SAP se conoce como "mandante". Un "mandante" o "cliente", en terminología SAP y desde el punto de vista funcional, se define como una unidad independiente del ERP que tiene sus propios datos y características (tipo de moneda, nombre de empresa, fiscalidad, productos etc.).

Por otro lado, en la web pública de Odoo correspondiente al "programa educativo" se creó una instancia inicial configurada por el profesor con el mismo conjunto de datos específicos que los de la instancia local.

Cada alumno crea su propia instancia del ERP en la nube a partir de la instancia del "programa educativo" con los mismos datos específicos, es decir, con los mismos productos, inventarios, pedidos de venta etc.

El uso de una "instancia" por alumno permite que el alumno modifique los datos de su sistema, por ejemplo, ordenando la reposición de un producto cuyo inventario es insuficiente sin afectar a los datos de inventario del resto de los alumnos en sus sistemas (instancias).

El ejercicio consiste en acceder a la base de datos del ERP para extraer una serie de datos y confeccionar un informe a medida sobre productos que hay pendiente de envío a 
determinados clientes. Como por cuestiones de privilegios no es posible el acceso a la base de datos de las instancias en la nube, el acceso se realiza a una base de datos del ERP instalado en local.

Para esto, se utiliza una herramienta de administración de SGBD denominada DbVisualizer (Free edition) instalada en la plataforma de cada alumno. Con esta herramienta, cada alumno extrae los datos de interés, mediante una consulta SQL y posteriormente se vuelcan a una hoja Excel con objeto de generar el informe a medida con dichos datos.

En la Fig. 2 se muestra un esquema de la arquitectura. Cada alumno accede con su herramienta de administración al SGBD de la plataforma local, el cual contiene los mismos datos que el SGBD de la plataforma en la nube. De este modo extrae los datos para confeccionar su informe el cual no está disponible como informe predefinido en el sistema ERP de la nube.

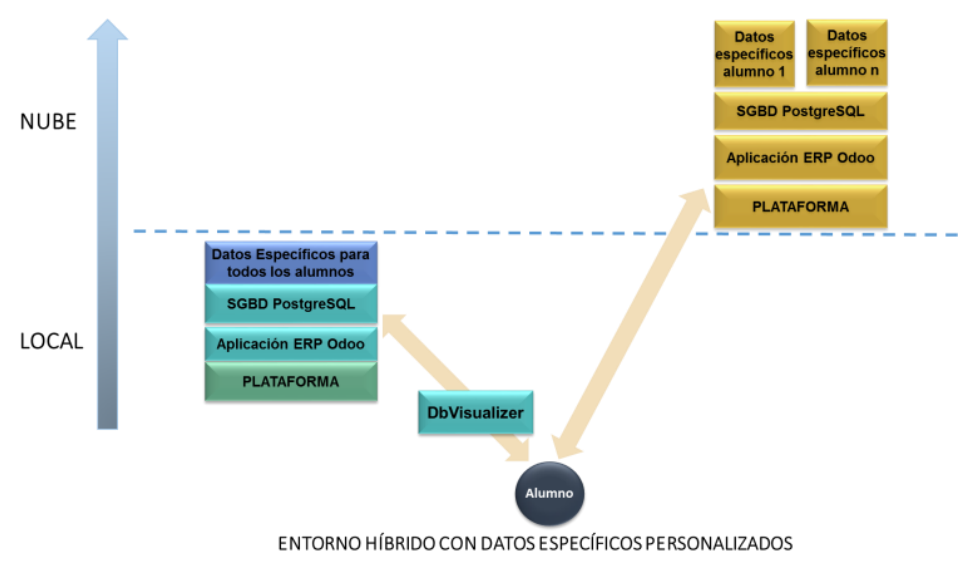

Fig. 2 Arquitectura híbrida de la práctica docente. Fuente: Elaboración propia

\section{Conclusiones}

El diseño de actividades prácticas efectivas con SIG, requiere disponer de software y datos con los que crear casos y escenarios en los que el alumno pueda desarrollar habilidades y enfrentarse a situaciones similares a la realidad empresarial.

Esto puede ser costoso y laborioso, por lo que una alternativa, es apoyarse en software abierto y gratuito disponible en la web. En la mayoría de las ocasiones, este software se adapta parcialmente a los objetivos docentes, por lo que es preciso integrarlo con otros sistemas instalados en plataformas locales con objeto de conseguir funcionalidades y experiencias de aprendizaje idóneas. 
Se plantean distintas arquitecturas para combinar SIG en la nube y en local, con las que diseñar actividades docentes adecuadas a objetivos curriculares que faciliten el aprendizaje del alumno.

Estas arquitecturas se centran en construir escenarios de utilidad educativa que cubran objetivos docentes específicos a partir de demos o software disponible en internet y aplicando distintos grados de personalización.

Con objeto de facilitar la confección de prácticas de laboratorio combinando sistemas disponibles en internet y sistemas implantados localmente, se concluye que la arquitectura de diseño de una práctica de laboratorio, viene determinada por la localización del SIG, nube o local, lo cual condicionará el grado de control y personalización que se tendrá sobre la aplicación y los datos.

La inmersión en un entorno SIG como el planteado, además de mejorar el conocimiento de los estudiantes sobre áreas de administración de empresa (contabilidad, logística, fabricación etc.), aporta un contexto significativo para el desarrollo de habilidades en la toma de decisiones y la resolución de problemas de gestión empresarial.

\section{Referencias}

Adams Becker, S. Cummins, M., Davis, A., Freeman, A., Hall Giesinger, C., \& Ananthanarayanan, V. (2017). NMC Horizon Report: 2017 Higher Education Edition (The New Media Consortium, ed.). Austin, Texas.

Caro, S., \& Reyes, J. (2003). Prácticas docentes que promueven el aprendizaje activo en ingeniería civil. Revista de Ingeniería, https://doi.org/http://dx.doi.org/10.16924\%2Friua.v0i18.479

Churchill, D., King, M., \& Fox, B. (2013). Learning design for science education in the 21st century. Journal of the Institute for Educational Research, 2(45), 404-421.

Ecology, I., \& For, S. I. (2017). Examples of graphs and tables generated in OMAT.

González Ruiz, C. J., Martín Gómez, S., \& Vega Navarro, A. (2018). Portales educativos: la producción de materiales didácticos digitales. @Tic. Revista D'Innovació Educativa, (20), 89. https://doi.org/10.7203/attic.20.12139

Marcos Ortega, J., de Borja Varona, F., \& Manuel Lopez, J. (2013). A Proposal of Computer Practical Clases related to Reinforced Concrete Structures for Civil Engineering and Architecture Degress. In I. Chova, LG and Martinez, AL and Torres (Ed.), EDULEARN13: 5th International Conference on Education and New Leartning Technologies (pp. 559-568).

Odoo SA. (2019). Prueba Odoo Online. Retrieved from https://www.odoo.com/es_ES/trial

SAP AG. (2019). Pruebas gratuitas de software. Retrieved from https://www.sap.com/spain/products/free-trials.html 